\title{
Joint Topology Optimization, Power Control and Spectrum Allocation for Intra-Vehicular Multi-hop Sensor Networks using Dandelion-encoded Heuristics
}

\author{
Javier Del Ser ${ }^{1,2}$, Miren Nekane Bilbao ${ }^{2}$, Cristina Perfecto ${ }^{2}$, Antonio \\ Gonzalez-Pardo ${ }^{3}$, and Sergio Campos-Cordobes ${ }^{1}$ \\ 1 TECNALIA. OPTIMA Unit, E-48160 Derio, Spain, \\ \{javier.delser, sergio.campos\}@tecnalia.com \\ 2 University of the Basque Country UPV/EHU, 48013 Bilbao, Spain, \\ \{javier.delser, nekane.bilbao, cristina.perfecto\}@ehu.eus \\ 3 Basque Center for Applied Mathematics (BCAM), 48009 Bilbao, Spain, \\ agonzalezp@bcamath.org
}

\begin{abstract}
In the last years the interest in multi-hop communications has gained momentum within the research community due to the challenging characteristics of the intra-vehicular radio environment and the stringent robustness imposed on critical sensors within the vehicle. As opposed to point-to-point network topologies, multi-hop networking allows for an enhanced communication reliability at the cost of an additional processing overhead. In this context this manuscript poses a novel bi-objective optimization problem aimed at jointly minimizing 1 ) the average Bit Error Rate (BER) of sensing nodes under a majority fusion rule at the central data collection unit; and 2) the mean delay experienced by packets forwarded by such nodes due to multi-hop networking, frequency channel switching time multiplexing at intermediate nodes. The formulated paradigm is shown to be computationally tractable via a combination of evolutionary meta-heuristic algorithms and Dandelion codes, the latter capable of representing tree-like structures like those modeling the multi-hop routing approach. Simulations are carried out for realistic values of intra-vehicular radio channels and co-channel interference due to nearby IEEE 802.11 signals. The obtained results are promising and pave the way towards assessing the practical performance of the proposed scheme in real setups.
\end{abstract}

Keywords: Intra-vehicular networks; Routing; Spectrum Allocation; Dandelion Encoding; Evolutionary Meta-heuristics

\section{Introduction}

The last decade has witnessed a number of technological advances in shortrange wireless networking as a consequence of impending research efforts towards improving their efficiency in terms of energy consumption, resource usage and 
self-organization capabilities. Evidences abound, ranging from newly developed protocols and stacks to the practical assessment of their performance in different communication environments. Consequently, a plethora of application scenarios have been shown to potentially benefit from the inherent advantages of wireless networks (i.e. ease of deployment, less infrastructure needed, increased mobility and lower costs [1]).

The automotive sector is one of the application scenarios where wireless technologies have been extensively studied as a lightweight alternative to sense different constituent parts of the vehicle [2-5]. Surprisingly it has not been until recently $[6,7]$ when multi-hop wireless networking has been hypothesized as an effective communication option to cope with the stringent fading statistics of the intra-vehicular channel, whose lack of frequency selectivity impedes the adoption of adaptive spectrum shaping techniques $[8,9]$. Such challenging radio conditions get even more involved with the presence of outer, non-controllable interfering sources whose transmitted signals collide with that forwarded by the deployed vehicular sensors to the central on-board unit. For instance, many contributions have analyzed the behavior and resilience of IEEE 802.15.4 (Zigbee) sensors under interfering IEEE 802.11 signals in both general (i.e. application agnostic) setups $[10,11]$ and specific vehicular environments $[12,13]$.

Indeed, multi-hop networking has attracted most of the recent literature on intra-car wireless communications. To the knowledge of the authors studies so far have gravitated on proving the feasibility of multi-hop networks by applying well-established protocols to controlled intra-vehicular communication setups. The most notable example is the work in [14], where the so-called Collection Tree Protocol was utilized in its naïve form to verify the predicted gains of multi-hop intra-vehicular wireless networking. Although resource allocation has been recently studied for the intra-vehicular environment [15], spectrum coordination, topology and power control has not been yet jointly tackled for this specific communication scenario, even though the underlying radio particularities - i.e. strong fading characteristics, eventual spectrum interferers - and the spectrum flexibility of avant-garde short-range wireless sensors [16] call for further investigation on this topic.

This manuscript elaborates on the above noted lack of research by proposing a centralized bio-inspired scheme for optimally 1) routing the information captured by the compounding nodes of an intra-vehicular wireless network; 2) allocating the power utilized by each of such nodes; and 3) selecting the spectrum channel between each pair of sensors. This work builds upon the number of multi-channel short-range wireless transceivers made commercially available in recent times, such as the GP712 single radio multi-protocol chipset developed by GreenPeak Technologies [17]. By exploiting the a priori knowledge of the propagation statistics between sensors (which can be obtained by aside channel estimation techniques), the proposed scheme resorts to a combination of evolutionary meta-heuristics and a tree encoding approach to balance two conflicting objectives: the overall delay of the captured information due to propagation, multiplexing and channel access, and the bit error rate averaged over all sensors 
when their information is sent over multiple paths and fused at the on-board central unit (OBU). Experiments over realistically modeled intra-vehicular communication scenarios are discussed towards evincing the satisfactory performance of the proposed resource allocation algorithm.

The remainder of the manuscript is structured as follows: Section 2 poses the optimization problem to be tackled. Section 3 delves into the design of the proposed resource allocation algorithm. Experimental results are analyzed in Section 4 and finally, Section 5 concludes the paper.

\section{System Model and Problem Formulation}

As often made in the literature an intra-vehicular network can be conceptualized as a directed graph defined by the ordered pair $\mathcal{G} \doteq(\mathcal{V}, \mathcal{E})$, where $\mathcal{V}$ denotes the set of vertices or nodes compounding the network and $\mathcal{E}$ represents the set of edges or links, each connecting a given pair of nodes. Let $N \doteq|\mathcal{E}|$ and $M \doteq|\mathcal{V}|$, with $|\cdot|$ standing for set cardinality. Point-to-point communication over each link $e \in \mathcal{E}$ is established over a certain frequency channel $f(e) \in \mathcal{F} \doteq\left\{f_{1}, \ldots, f_{F}\right\}$, where $F$ is the number of available radio channels (e.g. $F=15$ for Zigbee [18]). On the other hand, each node $v \in \mathcal{V}$ transmits at a certain power $p(v) \in$ $\mathbb{R}\left[0, P_{\text {max }}\right]$, where it is assumed that the radio transceiver is capable of adjusting its utilized power at any intermediate value within that given range.

The link between node $v_{i}$ and $v_{j}$ (with $j \in\{1, \ldots, i-1, i+1, \ldots, N\}$ ) undergoes degradation due to the fading statistics between different constituent parts of the network. In this context, measurements reported and analyzed in [8] showed that the whole intra-vehicular wireless medium features frequencyflat signal fading, with different Line-Of-Sight (LOS) and Non-Line-Of-Sight (NLOS) propagation characteristics depending on the physical location of the transmitting sensor and the receiving node. As a consequence, the degradation suffered by the above link - hereafter denoted as $e_{i, j}$ - imprints a Bit Error Rate $\operatorname{BER}\left(v_{i}, v_{j}\right)$ on the transmitted signal. This error rate can be expressed as a closed-form expression depending on the Signal to Interference and Noise Ratio $\operatorname{SINR}\left(v_{i}, v_{j}\right)$ over such a link, which in turn is fixed by the transmit power $p\left(v_{i}\right)$ and the signals interfering on receiver $v_{j}$ over the utilized frequency $f\left(e_{i, j}\right)$. For instance, in the IEEE 802.15.4-2006 standard utilized by Zigbee [18, Section E.4.1.8] $\operatorname{BER}\left(v_{i}, v_{j}\right)$ can be approximated by

$$
\operatorname{BER}\left(v_{i}, v_{j}\right) \doteq \operatorname{BER}\left(e_{i, j}\right) \approx \frac{1}{30} \sum_{n=2}^{16}(-1)^{n}\left(\begin{array}{c}
16 \\
n
\end{array}\right) e^{\frac{20(1-n) \operatorname{SinR}\left(v_{i}, v_{j}\right)}{n}},
$$

where effects from fading and interfering sources are usually included as summing contributions in $\operatorname{SINR}\left(v_{i}, v_{j}\right)$, i.e.

$$
\operatorname{SINR}\left(v_{i}, v_{j}\right)=\frac{p\left(v_{i}\right) \cdot L\left(v_{i}, v_{j}\right)}{\sum_{\substack{i^{\prime}=1 \\ i^{\prime} \neq i, j}}^{M} p\left(v_{i^{\prime}}\right) L\left(v_{i^{\prime}}, v_{j}\right) \mathbb{I}\left[f\left(e_{i^{\prime}, j}\right)=f\left(e_{i, j}\right)\right]+\left(N_{0} / 2\right) \Delta_{f\left(e_{i, j}\right)}},
$$


with $L\left(v_{i}, v_{j}\right)$ comprising fading/scattering channel losses from node $v_{i}$ to node $v_{j} ; \Delta_{f}$ represents the bandwidth of channel $f \in \mathcal{F} ; \mathbb{I}[\cdot]$ is an auxiliary function taking value 1 if its argument is true (and 0 otherwise); and $N_{0}$ is the noise power spectral density measured in watts per hertz. Loss factors also depend $\operatorname{logarithmically}$ on the distance between nodes $v_{i}$ and $v_{j}$ via a loss exponent $\gamma_{i, j}$.

Following the diagram depicted in Figure 1, each compounding sensor of the intra-vehicular network forwards their captured information to an on-board central receiver (coined previously as OBU) through a multi-hop tree-like route. By a slight notational abuse, each of such routes will be denoted as a subset $\mathcal{E}_{i}=\left\{e_{1}^{i}, e_{2}^{i}, \ldots, e_{N_{i}}^{i}\right\} \subseteq \mathcal{E}$, which includes all such $N_{i} \doteq\left|\mathcal{E}_{i}\right|$ edges in the network graph that participate in the route from node $v_{i}$ to the OBU. Since this work focuses on tree topologies, it should be obvious that $\left|\cup_{i=1}^{M} \mathcal{E}_{i}\right|=M$, i.e. the number of total links compounding the multi-hop route is equal to the overall number of nodes in the network. If a decode-and-forward relying approach is assumed, the bit error rate at the OBU for the information sent by node $v_{i}$ will be given, for odd $N_{i}$, by

$$
\begin{array}{r}
\operatorname{BER}_{\circledast}\left(v_{i}\right)=\sum_{x=1}^{N_{i}} B E R\left(e_{x}^{i}\right) \prod_{\substack{y=1 \\
y \neq x}}^{N_{i}}\left(1-\operatorname{BER}\left(e_{y}^{i}\right)\right) \\
+\sum_{x=1}^{N_{i}} B E R\left(e_{x}^{i}\right) \sum_{\substack{y=1 \\
y \neq x}}^{N_{i}} B E R\left(e_{y}^{i}\right) \sum_{\substack{z=1 \\
z \neq x, y}}^{N_{i}} B E R\left(e_{z}^{i}\right) \prod_{\substack{k=1 \\
k \neq x, y, z}}^{N_{i}}\left(1-\operatorname{BER}\left(e_{k}^{i}\right)\right) \\
+\ldots+\sum_{x=1}^{N_{i}}\left(1-B E R\left(e_{x}^{i}\right)\right) \prod_{\substack{y=1 \\
y \neq x}}^{N_{i}} B E R\left(e_{y}^{i}\right),
\end{array}
$$

i.e. as the probability that an odd number of links within $\mathcal{E}_{i}$ incurs in error (a similar expression can be obtained for even $N_{i}$ ). The average Bit Error Rate of the intra-vehicular sensor network when operating on the relying routes specified by $\left\{\mathcal{E}_{i}\right\}_{i=1}^{M}$ will be hence expressed as

$$
\mathrm{BER}_{\circledast}^{a v g} \doteq \frac{1}{M} \sum_{i=1}^{M} \operatorname{BER}_{\circledast}\left(v_{i}\right),
$$

which can be conceived as an overall measure of the communication quality of the sensor network deployed inside the vehicle.

The interference caused among nodes within the network and the eventual presence of external, non-controllable radio sources capable of creating cochannel interference in the same spectral band (e.g. IEEE 802.11 and Zigbee) can be mitigated by overlapping $\Psi$ redundant multi-hop routes that transmit the same information to the central OBU, which fuses the received flows under a given fusion criteria. One of such criteria is the so-called majority voting, which decides for the value of the $k$-th bit $b_{k}^{i}$ sent by node $i$ over each overlaid 


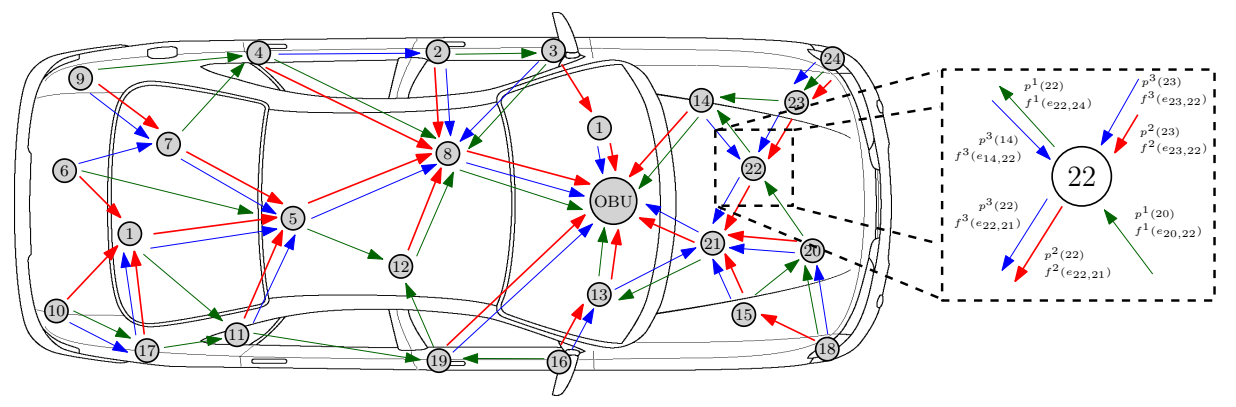

Fig. 1. Diagram representing the scenario tackled in this manuscript for $\Psi=3$ multihop routes overlaid over $M=24$ nodes. Each colored route can be modeled as a tree graph whose error and delay performance for every compounding node depends not only on the topology itself, but also on interfering signals from nearby nodes.

multi-hop route $\left\{\mathcal{E}_{i}^{\psi}\right\}_{i=1}^{M}$ as

$$
b_{k}^{i}= \begin{cases}1 & \text { if } \sum_{\psi=1}^{\Psi} b_{k}^{\psi} \geq \Psi / 2, \\ 0 & \text { if } \sum_{\psi=1}^{\Psi} b_{k}^{\psi}<\Psi / 2 .\end{cases}
$$

If by a similar development to Expression (2) we denote the Bit Error Rate for sensor $i$ over multi-hop route $\left\{\mathcal{E}_{i}^{\psi}\right\}_{i=1}^{M}$ as $\operatorname{BER}_{\circledast}^{\psi}\left(v_{i}\right)$, the overall error obtained for sensor $i$ at the OBU under majority voting $(m v)$ is given, for odd $\Psi$, by

$$
\operatorname{BER}_{\circledast}^{m v}\left(v_{i}\right) \doteq \sum_{z=\lceil\Psi / 2\rceil}^{\Psi}\left(\sum_{\mathcal{M} \in \mathcal{M}^{z}}\left(\prod_{i \in \mathcal{M}} \operatorname{BER}_{\circledast}^{\psi}\left(v_{i}\right) \prod_{i^{\prime} \in \mathcal{V} / \mathcal{M}}\left(1-\operatorname{BER}_{\circledast}^{\psi}\left(v_{i^{\prime}}\right)\right)\right)\right),
$$

where $\mathcal{M}^{z}$ denotes the set of all $z$-length combinations drawn from the integer set $\{1, \ldots, M\}$. By averaging over the whole set of nodes an overall measure of the communication robustness of the whole network can be obtained as

$$
\operatorname{BER}_{\circledast}^{a v g, m v}=\frac{1}{M} \sum_{i=1}^{M} \operatorname{BER}_{\circledast}^{m v}\left(v_{i}\right),
$$

which should be minimized by properly optimizing the values of its controlling variables $\left\{p^{\psi}\left(v_{i}\right)\right\}_{i=1}^{M}$ (power), $\left\{f^{\psi}\left(e_{i, j}\right)\right\}_{i, j=1}^{M}$ (frequency channel between nodes) and the topology of each of the different multi-hop routes. For notational convenience edges belonging to the tree graph modeling each of the overlaid multi-hop routes connecting all nodes to the OBU will be denoted as $\left\{e_{i, j}^{\psi}\right\}_{i, j=1}^{N}$.

Unfortunately the minimization of the above error comes along with a delay penalty due to 1 ) the switch of frequency channels at every transmitting node; and 2) the time multiplexing of these flows in intermediate nodes receiving data from different nodes and forwarded over redundant routes. As for the former the incurred delay is assumed to increase linearly with the different number 
of channels to be heard by every node. Specifically, if the time taken by the radio interface to shift from one channel to another is denoted by $T_{s w}$ and no parallelization between reception and transmission tasks is assumed, the worstcase switching delay at node $v_{j}$ and multi-hop route $\left\{\mathcal{E}_{i}^{\psi}\right\}_{i=1}^{M}$ will be given by

$$
D_{s w}^{\psi}\left(v_{j}\right)=T_{s w}\left(\left|\left\{f\left(e_{i, j}^{\psi}\right)\right\}_{\substack{i=1 \\ i \neq j}}^{N}\right|+\left|\left\{f\left(e_{j, n}^{\psi}\right)\right\}_{\substack{n=1 \\ n \neq j}}^{N}\right|-2\right),
$$

i.e. as the switching delay times the number of unique frequency channels used for the links incoming at - and outgoing from - node $v_{j}$. The second term of the overall delay will assume all nodes operate in saturation regime, which yields a multiplexing time linearly dependent on the number of links to be relayed and the estimated time of the utilized communication stack for transmitting a packet. In Zigbee this time reduces to a sum of the time-on-air of the packet at hand and the time for CSMA-CA channel access and eventual retries. For instance, for the XBee commercial radio module [19], 16-bit addressing, 127-byte payload and clear channel access, this latency is

$$
D_{m x}^{\psi}\left(v_{i}\right)=0.001 \cdot\left(0.544+\left(0.032 \cdot 127 \cdot\left|\left\{e_{i, j}^{\psi}\right\}_{\substack{j=1 \\ j \neq i}}^{N}\right|\right)\right) \text { (in seconds). }
$$

The information sent by every node undergoes accumulated delays at intermediate nodes due to the above two terms. As a result the overall, worst-case delay for the information sent by node $v_{i}$ will be given by

$$
D_{\circledast}^{\psi}\left(v_{i}\right)=\sum_{j \in \mathcal{M}_{i \rightarrow O B U}^{\psi}}\left(D_{m x}^{\psi}\left(v_{j}\right)+D_{s w}^{\psi}\left(v_{j}\right)\right),
$$

where $\mathcal{M}_{i \rightarrow O B U}^{\psi}$ denotes the subset of nodes that participate in the path from node $v_{i}$ (included) to the OBU. From this expression the overall delay metric averaged over all nodes in the network can be computed under two different assumptions: if all nodes await for the completion of all transmissions over a certain multi-hop route $\left\{\mathcal{E}_{i}^{\psi}\right\}_{i=1}^{M}$ before proceeding with the next one, the overall delay will be fixed for all nodes and given by

$$
D_{\circledast}^{\text {avg }}=\sum_{\psi=1}^{\Psi} \max _{i \in\{1, \ldots, M\}} D_{\circledast}^{\psi}\left(v_{i}\right)
$$

which, in what regards to the allocation of resources, can be conceived as a set of independent routes. On the contrary, if transmissions over different routes are allowed to hold concurrently in the network, nodes will require switching frequency channels and relay links belonging to different routes. This implies that the overall worst-case delays in Expressions (7) and (8) are modified to

$$
\begin{aligned}
& D_{s w}\left(v_{j}\right)=T_{s w}\left(\left|\left\{\left\{f\left(e_{i, j}^{\psi}\right)\right\}_{\substack{i=1 \\
i \neq j}}^{N}\right\}_{\psi=1}^{\Psi}\right|+\left|\left\{\left\{f\left(e_{j, n}^{\psi}\right)\right\}_{\substack{n=1 \\
n \neq j}}^{N}\right\}_{\psi=1}^{\Psi}\right|-2\right), \\
& D_{m x}\left(v_{i}\right)=0.001 \cdot\left(0.544+\left(0.032 \cdot 127 \cdot\left|\left\{\left\{e_{i, j}^{\psi}\right\}_{\substack{j=1 \\
j \neq i}}^{N}\right\}_{\psi=1}^{\Psi}\right|\right)\right),
\end{aligned}
$$


giving rise to an overall average delay given by

$$
D_{\circledast}^{a v g}=\frac{1}{M} \sum_{i=1}^{M} \max _{\psi \in\{1, \ldots, \Psi\}}\left(\sum_{j \in \mathcal{M}_{i \rightarrow O B U}^{\psi}}\left(D_{m x}\left(v_{j}\right)+D_{s w}\left(v_{j}\right)\right)\right),
$$

which depends roughly on the transmitted power, frequency and topology of the constructed overlay routes. With these definitions in mind, the problem tackled in this paper can be formulated as finding an optimal allocation $\left\{p^{\psi}\left(v_{i}\right)\right\}_{i=1}^{M}$

(power), $\left\{f^{\psi}\left(e_{i, j}\right)\right\}_{i, j=1}^{M}$ (frequency channel between nodes) and $\left\{e_{i, j}^{\psi}\right\}_{i, j=1}^{N}$ (tree topology) for a given number of overlaid routes $\Psi$ such as the conflicting objectives defined in Expressions (6) (overall average BER) and (10) or (13) (overall delay) are simultaneously optimized, i.e.

$$
\left[\left\{p^{\psi}\left(v_{i}\right)\right\}_{i=1}^{M},\left\{f^{\psi}\left(e_{i, j}\right)\right\}_{\substack{i, j=1 \\ i \neq j}}^{M},\left\{e_{i, j}^{\psi}\right\}_{i, j=1}^{N}\right]_{\psi=1}^{\Psi}=\arg \min \left[\mathrm{BER}_{\circledast}^{a v g, m v}, D_{\circledast}^{a v g}\right],
$$

subject to

$$
\begin{array}{r}
p^{\psi}\left(v_{i}\right) \in\left[0, P_{\max }\right] \forall i \in\{1, \ldots, M\}, \\
f^{\psi}\left(e_{i, j}\right) \in \mathcal{F}=\left\{f_{1}, \ldots, f_{F}\right\} \forall e_{i, j} \in \mathcal{E},
\end{array}
$$

and the additional constraint that routes $\left\{e_{i, j}^{\psi}\right\}_{i, j=1}^{N} \forall \psi \in\{1, \ldots, \Psi\}$ can be modeled as a tree graph spanning the whole set of nodes within the network and rooted on the OBU.

Due to the complexity of the problem and the conflicting nature of both objectives, this work proposes to resort to multi-objective evolutionary metaheuristic algorithms aimed at estimating the Pareto front differently trading one objective for the other. This approach requires efficient solution encoding strategies capable of jointly representing the tree topology of the multi-hop routes, the power and the frequency channel utilized by the nodes for each route as will be explained in detail in the following section.

\section{Proposed Resource Allocation Algorithm}

The ultimate purpose of the resource allocation algorithm proposed in this paper is to estimate the set of values for the optimization problem posed in Expressions (14) to (16) optimally trading bit error rate performance for average delay from the nodes to the central OBU. To this end a bio-inspired, population-based, multi-objective meta-heuristic approach simultaneously comprising power, frequency and topology optimization will be next described, including the solution encoding, its constituent operators and overall operation strategy.

\subsection{Solution Encoding}

All variables are represented by a vector $\mathbf{X}(k)$, with $k \in\{1, \ldots, K\}$ denoting the index of the solution within the population. This vector will contain the power 
(one value per node and route, $M \Psi$ in total), frequency channel (one value per link and route, and since in every tree the number of links coincides with the number of sensors, $M \Psi$ in total) and an integer representation of the tree topology of every route. As for the latter our approach will resort to the family of Dandelion-like codes, a subclass of the general category known as Cayley codes. A Cayley code is essentially a bijection between the set of all labeled unrooted trees on $M$ nodes and $M-2$ tuples of node labels, i.e. each tree corresponds to a unique Cayley code and vice versa. Therefore, the overall solution vector will be given by $\Psi \cdot(3 \cdot M-2)$ integer values.

While the variable encoding for the transmit power and frequency per link is straightforward, the rationale for selecting Dandelion codes for representing the multi-hop routes lies on their full coverage, zero-bias and perfect closure, all desirable properties for ensuring efficient encoding/decoding and a bounded locality when perturbed via evolutionary operators. In fact, Dandelion codes have been shown to possess better locality and heritability characteristics than any other Cayley codes when undergoing different evolutionary operators [20, 21]. This superior performance of Dandelion codes has unleashed a flurry of research gravitating on their application in different scenarios and sectors, such as Telecommunications [22] and Energy [23].

\subsection{Meta-heuristic Solver}

With the previous solution encoding approach in mind, a meta-heuristic algorithm is required to evolve the solution population towards regions of progressively increased Pareto optimality. This will be accomplished by the constituent operators of the so-called Harmony Search (HS) meta-heuristic algorithm, first presented in [24] and since then proven to perform statistically better than other meta-heuristic schemes in a wide variety of applications [25]. This algorithm inspires from the collaborative behavior of musicians when improvising aesthetically good harmonies; in fact the compounding operators of HS can be regarded as computationally modeled behavioral patterns commonly observed in music composition. At this point the authors would like to point out that despite the controversy around this algorithm in regards to its similarity to a special case of $(\mu+1)$ Evolutionary Strategies [26,27], in this paper we will use the HS notation in impartial conformity with the majority of the related literature.

Following the above simile solutions are referred to as harmonies in the context of HS. Likewise, the population of potential solutions as harmony memory or HM. This memory undergoes a set of intelligent operators repeatedly until a stop criterion is satisfied. HS applies these operators on a per-note basis with statistical independence between nodes, which ultimately permits to balance more effectively the intensification and diversification of the underlying search procedure. When particularized to the problem tackled in this paper, the nominal search process of the HS algorithm breaks down into four steps, schematically depicted in Figure 2 and described as follows: 
1. Initialization of the HM: since no a priori information will be assumed, the HM is populated with harmonies whose individual elements are drawn uniformly at random from their corresponding alphabets. Without loss of generality other initialization criteria can be used such as e.g. a distance-based minimum spanning tree for the notes representing the topology of the routes.

2. Improvisation: after initializing the set of stored solutions, a new harmony memory $\{\widehat{\mathbf{X}}(k)\}_{k=1}^{K}$ is improvised. For each note in $\{\mathbf{X}(k)\}_{k=1}^{K}$ the following operators are subsequently applied:

- The Harmony Memory Considering Rate (HMCR) is driven by the probabilistic parameter $\vartheta \in[0,1]$, and establishes the probability that the new improvised value for a given note is drawn uniformly from the values taken by the same note in the $K-1$ remaining harmonies. Otherwise

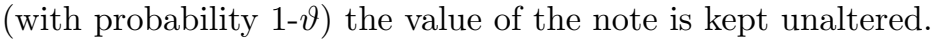

- The Pitch Adjustment Rate, controlled by the parameter $\varphi \in[0,1]$, sets the probability that the value for a given note is replaced with any of its neighborhood in the corresponding alphabet. Here the notion of neighborhood depends roughly on how the value of the fitness functions behaves over the alphabet with respect to its value for the note value of reference. In this work the alphabet for all variables will be sorted in natural ordering; while this criterion can be intuitively aligned with the expected impact of the transmit power on the objective functions, this intuition may not hold for those variables representing the frequency assignment and the topology of the multi-hop routes. Hence, in such variables this operator reduces to a uniform randomization of the value, similar to the naïve mutation operator in Genetic Algorithms.

3. Evaluation and update of the Harmony Memory: once the fitness values for every candidate harmony in the newly produced memory have been computed, this bi-objective solver selects the prevailing set of $K$ harmonies under a criterion based on rank and crowding distance: each improvised harmony $\widehat{\mathbf{X}}(k)$ is labeled with a numerical score depending on its Pareto dominance level with respect to the rest of individuals (both the other new harmonies and those remaining from the previous iteration). Once all individuals have been ranked in these terms, their crowding distance is computed and utilized as a secondary score to evaluate individuals within the same Pareto dominance level. Finally, those $K$ harmonies first ranked in terms of Pareto optimality (primary criterion) and crowding distance (secondary criterion) are kept for subsequent iterations.

4. Termination: a stopping criterion based on a maximum number of iterations is imposed to declare the HM as the best Pareto solution attained by the proposed scheme. If the criterion has not been met steps 2 to 4 are repeated.

As for the implementation of the above meta-heuristic algorithm, it is important to note that a high number of variables are optimized via a single fitness function. For instance, when dealing with $\Psi=3$ routes and $M=50$ nodes a total of $\Psi \cdot(3 \cdot M-2)=444$ variables are optimized by the above solver. In order to handle efficiently the possible counteractions between the variables over 


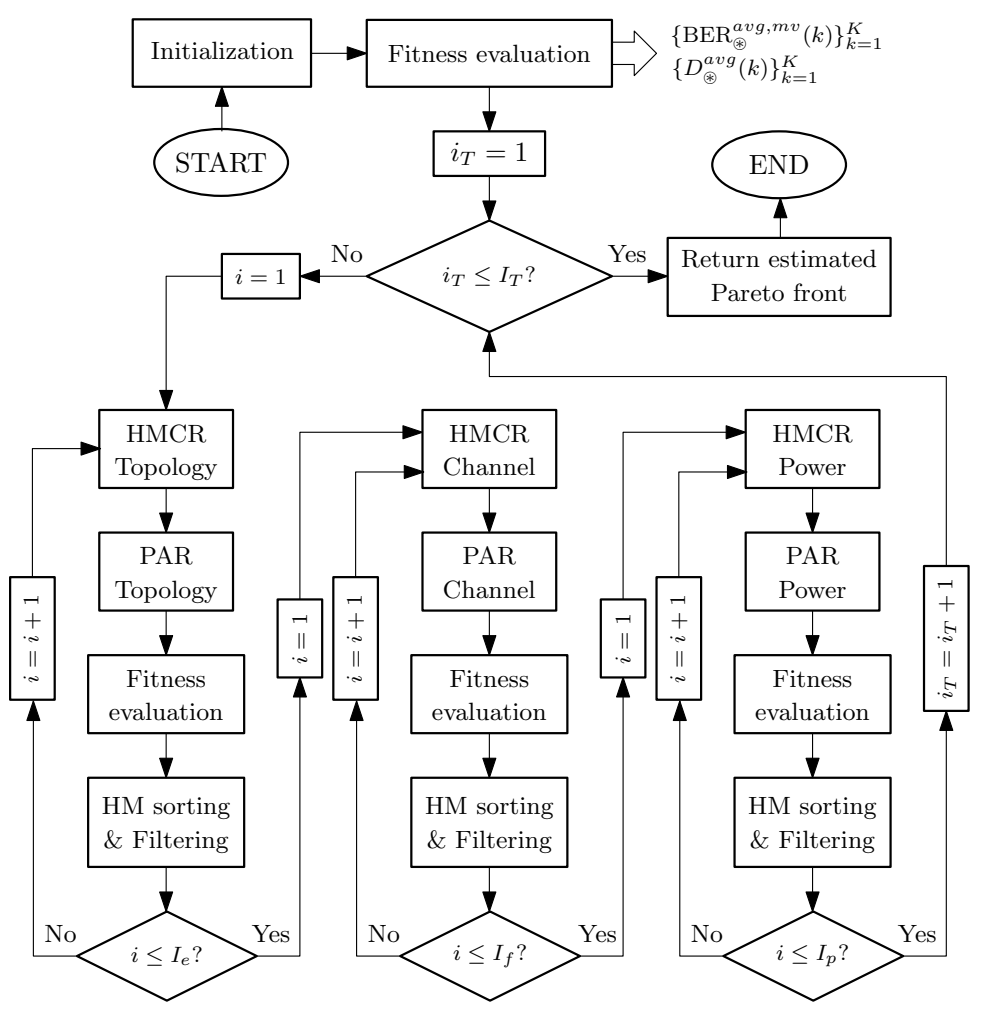

Fig. 2. Flow diagram of the proposed meta-heuristic resource allocation algorithm. HM sorting is performed based on Pareto rank and crowding distance criteria. It is important to note that the application of the HS operators is iterated over the resources to be allocated - topology, channel or power - during a number of iterations each.

the considered objective functions, the application of the constituent harmony search operators is iterated over each variable set - topology, spectrum channel and transmit power - during a certain number of iterations $I_{e}, I_{f}$ and $I_{p}$, respectively. This block-wise iterative procedure is then globally repeated for $I_{T}$ iterations, accounting up to a total of $I_{T} \cdot\left(I_{e}+I_{f}+I_{p}\right)$ fitness evaluations.

\section{Experiments and Results}

In order to assess the performance of the proposed resource allocation algorithm under different operational radio conditions, several scenarios have been designed and simulated by considering an intra-vehicular mesh of $M=75$ wireless sensors uniformly deployed at random inside the car. The central OBU has been assumed to be located in the front part of the vehicle. The maximum transmit power of every compounding node is $P_{\max }=0 \mathrm{dBm}$, whereas noise power spectral density is fixed to $N_{0}=-134 \mathrm{dBm} / \mathrm{Hz}$. Communications take place over an spectrum band divided in $|\mathcal{F}|$ channels of equal bandwidth (5 MHz each). 
Radio links established inside the vehicle are modeled as Rayleigh or Rician fading channel depending on whether source and destination nodes are located (Line Of Sight, LOS) or not (NLOS) inside the same vehicle compartment (i.e. trunk, cabin, in engine, under engine). The path loss exponent driving the exponential dependence of the transmit power on distance is set to $\gamma_{i, j}=3$ $\forall i, j \in M \times M$. An additional log-normal shadowing component with standard deviation equal to $8 \mathrm{~dB}$ is included in the large-scale fading model. Values for the ratio between the direct component and the variance of the multi-hop signal are set so as to reflect 1) the radio propagation complexity of each section of the car due to e.g. metallic parts; and 2) the boundaries between the aforementioned vehicle compartments. Figure 3.a and 3.b exemplify this channel modeling by depicting the average channel gain for a source sensor located in the trunk and inside the engine, respectively. The values adopted for the above radio parameters are suitable for modeling intra-vehicular wireless networks according to recent literature $[8,28]$.

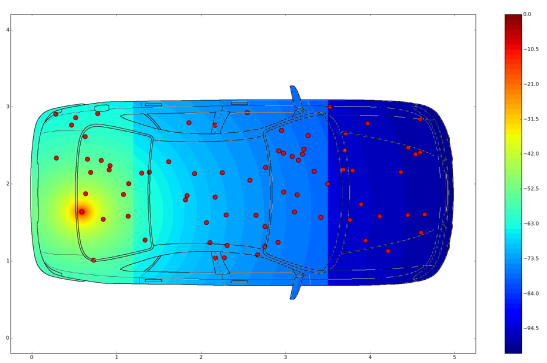

(a)

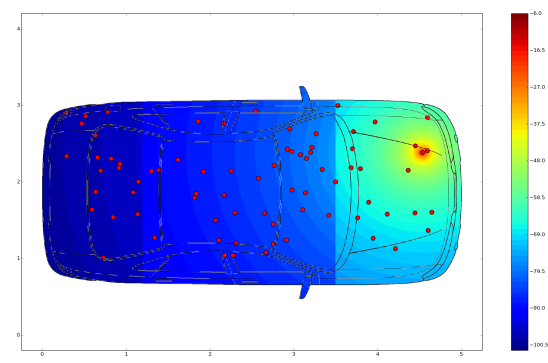

(b)

Fig. 3. Heatmap representing the average channel gain (in $\mathrm{dB}$ ) inside the vehicle for a sensor node transmitting (a) from the trunk; (b) from the outer part of the engine. Deployed nodes are depicted as red circles. The channels utilized in the simulation experiments incorporate log-normal shadowing. Color scales vary between figures.

Switching between two frequency channels is assumed to take $T_{s w}=2 \cdot 10^{-4}$ seconds. The parameters controlling the resource allocation algorithm are fixed to $K=100, \vartheta=0.7, \varphi=0.1, I_{e}=I_{f}=I_{p}=10$ and $I_{T}=10$, values that have been optimized via an off-line grid search (results not included in the manuscript for the sake of space). The algorithm has been simulated over two different scenarios so as to shed light on the single-shot performance of the proposed algorithm, which are discussed in what follows.

\section{Scenario A: Full Spectrum Availability}

In this case a total of $|\mathcal{F}|=16$ channels (as in physical stacks such as IEEE 802.15.4) are available. It is expected that the resource allocation algorithm has enough channel diversity to achieve the lowest interference level within the set of simulated scenarios. When combined with a suitable, interference-aware transmit power assignment over nodes, the Pareto front optimally balancing error rate 
and delay should connect maximal points characterized by 1) an underlying star network topology (minimum delay, maximum error rate); and 2) a tree-like network layout with short-range links (maximum delay, minimum error rate).

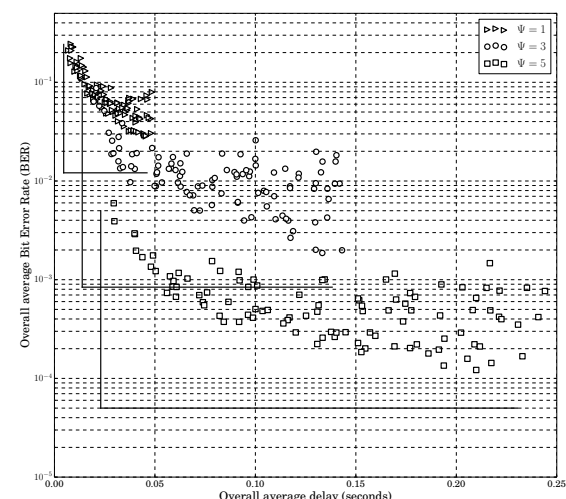

(a)
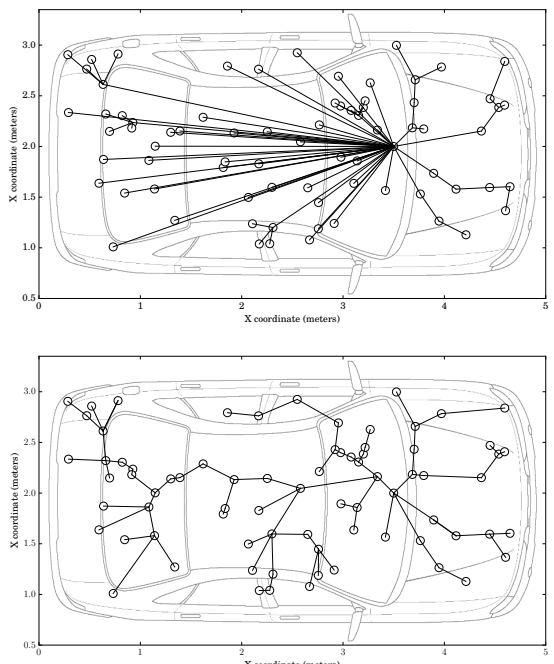

(c)

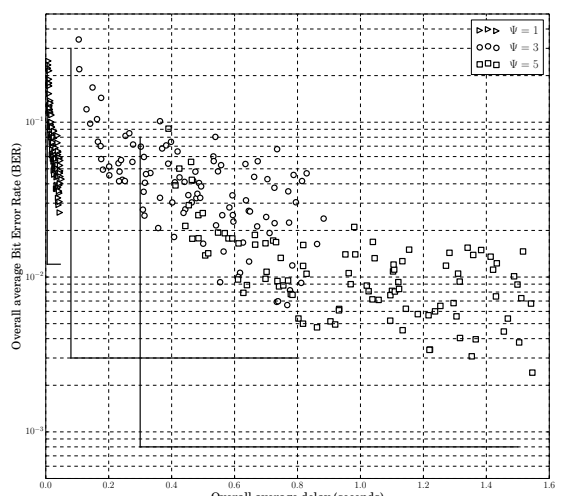

(b)
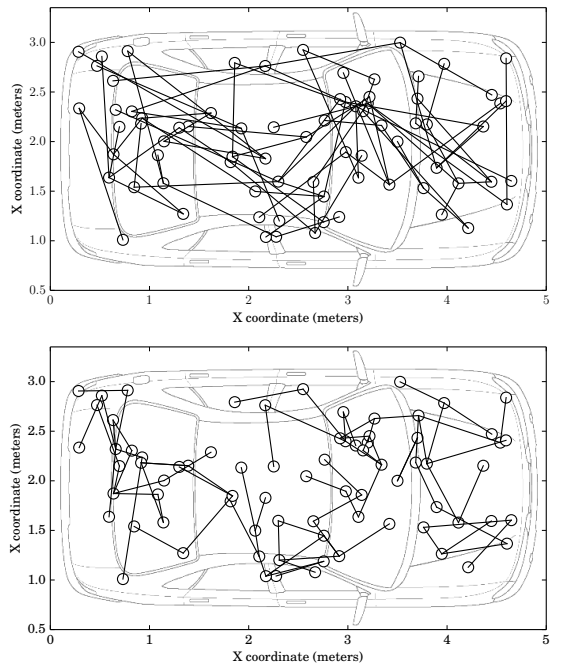

(d)

Fig. 4. Approximate Pareto fronts produced by the proposed resource allocation algorithm for different values of $\Psi$ and the delay model in Expressions (10) (a) and (13) (b). Figures (c) and (d) depict the network topology of a multi-hop route selected at random in the case with $\Psi=3$ for the extreme points of the corresponding fronts.

The obtained results verify our prior intuitions on the behavior of the algorithm under the two assumptions taken for the modeling of the overall delay. When nodes await for the completion of all transmissions within a multi-hop route $\left\{\mathcal{E}_{i}^{\psi}\right\}_{i=1}^{M}$, the results in Figure 4.a evince that the Pareto front approximation produced by the algorithm approaches several performance asymptotes estimated based on: 
- The majority fusion of $\Psi$ flows characterized by individual, interference-free error rates for each node.

- The minimum delay per node attainable by star network topologies with no need for channel switching, i.e. $\min D_{\circledast}^{\text {avg }}=\Psi \cdot D_{m x}^{\psi}\left(v_{i}\right)$.

The good agreement of the estimated resource allocation algorithm with its expected behavior is also buttressed by the plots in Figure 4.c, which depicts the topology of a randomly chosen route produced by the algorithm at both extremes of the approximated Pareto front for $\Psi=3$. On the contrary, when modeling the delay under the assumption that transmissions belonging to different routes are concurrently routed at intermediate nodes, the objectives to be optimized undergo the effects of interference, frequency switching and multiplexing. The corresponding approximated Pareto fronts degrade accordingly, as Figure 4.b clearly shows. In regards to Figure 4.d, topologies associated to the extreme Pareto points in the $\Psi=3$ case of Figure 4.b do not resemble any longer a treelike or star network, the reason being that the allocation of resources becomes more involved due to the interactions between different multi-hop routes.

\section{Scenario B: Restricted Spectrum Availability}

In this second scenario the vehicle at hand is assumed to suffer from the interfering effects of an external IEEE 802.11 access point transmitting at $20 \mathrm{dBm}$ 7 meters away from the trunk. The access point is assumed to occupy different percentages $\Delta$ of the set of $|\mathcal{F}|$ spectrum channels available for intra-vehicular networking. We will restrict this second analysis to the results obtained for the simplistic delay model.

Results in Figure 5.a and 5.b reveal that the proposed algorithm reacts satisfactorily against the in-band interference imposed by the external access point. When focusing on low-delay allocation policies, the assigned resources do not vary significantly due to the localized effect of the interference at the receiving end which, in the case of star-like topologies, is mainly located at the OBU. By contrast, when focusing on resilient topologies, the network is built by shortlength links which, for the sake of minimizing inter-node interference, demand as many frequency channels as possible to provide enough spectral diversity among transmissions concurring at the same node.

As the interference from the external access point becomes dominant over the transmission band (i.e. as $\Delta$ increases) nodes located inside the trunk undergo a increasingly severe spectrum scarcity, thus their achievable error and delay figures degrade accordingly. In Figure 5.b it is important to observe that as the number of interference-free spectrum channel reduces, nodes inside the trunk are connected via physically longer radio links, which can be justified by the fact that the algorithm finds it better (in the strict sense of Pareto optimality) to minimize the number of intermediate hence relaying nodes. This is accomplished by making the network layout less deep and more central. 


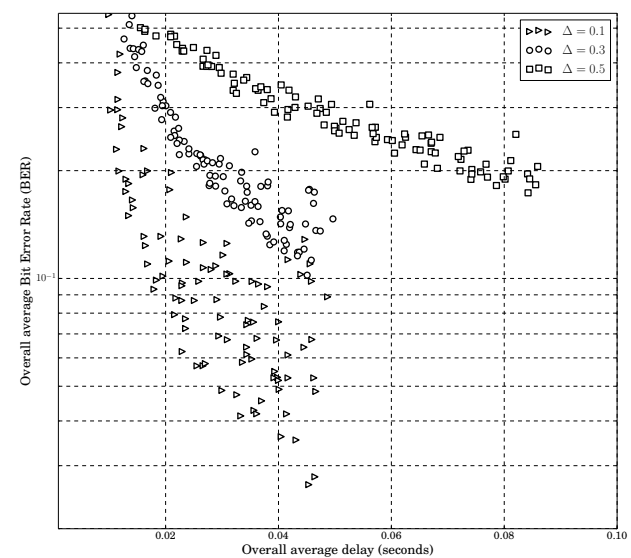

(a)
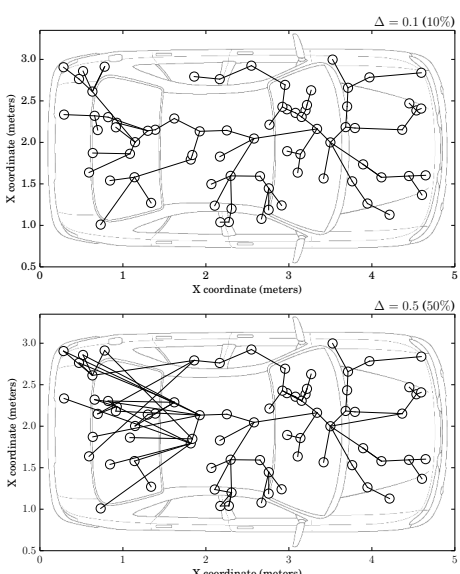

(b)

Fig. 5. (a) Approximate Pareto front and (b) network topology associated to the points with minimum overall bit error rate for the simplistic delay model in presence of a external IEEE 802.11 interferer over $\Delta \%$ of the utilized spectrum band.

\section{Conclusions and Future Research Lines}

This manuscript has elaborated on the simultaneous management of spectrum, topology and power in an intra-vehicular wireless network. The stringent radio propagation and interference characteristics of this radio environment and their impact on the optimal allocation of the aforementioned resources are mathematically formulated as a constrained bi-objective optimization problem jointly considering two conflicting criteria: bit error rate after majority data fusion and overall average latency undergone by the compounding nodes of the network. Intuitively, error rate performance is strongly affected by co-channel interference and fading statistics, which impose short-range communications and spectrum coordination between nearby nodes. On the other hand, latency is mainly due to switching among different spectrum channels and the service (queuing) time at intermediate nodes, which in contrast call for star-like networks with long-range links and scarce spectrum diversity.

The formulated problem aims at finding the set of Pareto-optimal resource allocation strategies that best trade one objective for the other. In order to cope with the inherent complexity of the problem evolutionary meta-heuristics have been utilized. The proposed approach resorts to the so-called Dandelion solution encoding scheme, which permits representing tree-like routes with maximum locality and heritability under evolutionary operators. In order to enhance the convergence speed of the overall solver, three separate yet interrelated search procedures are scheduled in practice, each operating on a single resource for a given number of iterations. The performance of the allocation method is assessed and discussed through experiments over a number of simulation scenarios, from 
which it is concluded that the proposed meta-heuristic scheme is capable to optimally allocate resources under distinct spectrum availability levels and external interfering signals.

Future research will be conducted towards including energy efficiency (i.e. minimization of the power consumption) as an objective in the formulated optimization problem. In addition, more realistic latency models will be considered by discarding any assumption on the transmission regime of the nodes. Finally, a performance benchmark between different evolutionary solvers (e.g. genetic algorithms) will be done in terms of Pareto optimality.

\section{Acknowledgments}

This work has been supported by the ARTEMIS Joint Undertaking and the Spanish Ministerio de Economia y Competitividad (DEWI project, ref1. 621353).

\section{References}

1. Kraemer, R., Katz, M. D.: Short-Range Wireless Communications: Emerging Technologies and Applications. John Wiley \& Sons (2009)

2. Lin, J. R., Talty, T., Tonguz, O. K.: On the Potential of Bluetooth Low Energy Technology in Vehicular Applications. IEEE Communications Magazine 53(1), 267-275 (2015)

3. Lu, N., Cheng, N., Zhang, N., Shen, X., Mark, J. W.: Connected Vehicles: Solutions and Challenges. IEEE Internet of Things Journal 1(4), 289-299 (2014)

4. Bas, C. U., Ergen, S. C.: Ultra-wideband Channel Model for Intravehicular Wireless Sensor Networks Beneath the Chassis: From Statistical Model to Simulations. IEEE Transactions on Vehicular Technology 62(1), 14-25 (2013)

5. Wu, X., Subramanian, S., Guha, R., White, R. G., Li, J., Lu, K. W., Bucceri, A., Zhang, T.: Vehicular Communications using DSRC: Challenges, Enhancements, and Evolution. IEEE Journal 1on Selected Areas in Communications 31(9), 399$408(2013)$

6. Hashemi, M., Si, W., Laifenfeld, M., Starobinski, D., Trachtenberg, A.: Intra-Car Multihop Wireless Sensor Networking: A Case Study. IEEE Communications Magazine 52(12), 183-191 (2014)

7. Ben Jaballah, W., Conti, M., Mosbah, M., Palazzi, C.E.: Fast and Secure Multihop Broadcast Solutions for Intervehicular Communication. IEEE Transactions on Intelligent Transportation Systems 15(1), 433-450 (2014)

8. Moghimi, A. R., Tsai, H.-M., Saraydar, C., Tonguz, O. K.: Characterizing IntraCar Wireless Channels. IEEE Transactions on Vehicular Technology 58(9), 52995305 (2009)

9. De Francisco, R., Huang, L., Dolmans, G., de Groot, H. Coexistence of zigbee wireless sensor networks and bluetooth inside a vehicle. IEEE International Symposium on Personal, Indoor and Mobile Radio Communications, 2700-2704 (2009)

10. Zhang, X., Shin, K. G. Enabling Coexistence of Heterogeneous Wireless Systems: Case for ZigBee and WiFi. Proceedings of the Twelfth ACM International Symposium on Mobile Ad Hoc Networking and Computing, 6 (2011).

11. Thonet, G., Allard-Jacquin, P., Colle, P.: ZigBee-WiFi Coexistence. Schneider Electric White Paper and Test Report, 1, 1-38 (2008) 
12. Lin, J.-R., Talty, T., Tonguz, O. K.: An Empirical Performance Study of Intravehicular Wireless Sensor Networks under WiFi and Bluetooth Interference. Proceedings of the IEEE Global Communications Conference (GLOBECOM) 581-586 (2013)

13. Tsai, H.-M., Tonguz, O. K., Saraydar, C., Talty, T., Ames, M., Macdonald, A.: Zigbee-based Intra-Car Wireless Sensor Networks: a Case Study. IEEE Wireless Communications 14(6), 67-77 (2007)

14. Hashemi, M., Si, W., Laifenfeld, M., Starobinski, D., Trachtenberg, A.: Intra-car Wireless Sensors Data Collection: A Multi-hop Approach. 2013 IEEE 77th Vehicular Technology Conference (VTC Spring), 1-5 (2013)

15. Sadi, Y., Ergen, S. C.: Optimal Power Control, Rate Adaptation and Scheduling for UWB-Based Intra-Vehicular Wireless Sensor Networks. IEEE Transactions on Vehicular Technology 62(1), 219-234 (2013)

16. Xu, R., Shi, G., Luo, J., Zhao, Z., Shu, Y.: Muzi: Multi-channel Zigbee Networks for Avoiding Wifi Interference. International Conference on Internet of Things and 4th International Conference on Cyber, Physical and Social Computing (iThings/CPSCom), 323-329 (2011)

17. GreenPeak Technologies press release: GreenPeak's new multi-channel chipset will simultaneously support ZigBee and Thread networks. Available at http://www . greenpeak.com/Press/ (retrieved on October 2015)

18. IEEE Standard 802.15.4-2006: Wireless Medium Access Control (MAC) and Physical Layer (PHY) Specifications for Low-Rate Wireless Personal Area Networks (WPANs) (2006)

19. Xbee module, http://www.digi.com/lp/xbee, Digi International Inc. Retrieved on October 2015.

20. Thompson, E., Paulden, T., Smith, D. K.: The Dandelion Code: A New Coding of Spanning Trees for Genetic Algorithms. IEEE Transactions on Evolutionary Computation 11(1), 91-100 (2007)

21. Perfecto, C., Bilbao, M. N., Del Ser, J., Ferro, A.: On the Heritability of Dandelionencoded Harmony Search Heuristics for Tree Optimization Problems. International Symposium on Innovations in Intelligent SysTems and Applications, 1-8 (2015)

22. Jafari, A. H., Lopez-Perez, D., Song, H., Claussen, H., Ho, L., Zhang, J. Small Cell Backhaul: Challenges and Prospective Solutions. EURASIP Journal on Wireless Communications and Networking 2015(1), 1-18 (2015)

23. Sabattin, J., Bolton, C. C., Arias, M., Parada, V.: Evolutionary Optimization of Electric Power Distribution using the Dandelion Code. Journal of Electrical and Computer Engineering 4 (2012)

24. Geem, Z. W., Kim, J. H., Loganathan, G. V.: A New Heuristic Optimization Algorithm: Harmony Search. Simulation 76(2), 60-68 (2001)

25. Manjarres, D., Landa-Torres, I., Gil-Lopez, S., Del Ser, J., Bilbao, M. N., SalcedoSanz, S., Geem, Z. W.: A Survey on Applications of the Harmony Search Algorithm. Engineering Applications of Artificial Intelligence 26(8), 1818-1831 (2013)

26. Weyland, D.: A Critical Analysis of the Harmony Search Algorithm - How not to Solve Sudoku. Operations Research Perspectives 2, 97-105 (2015)

27. Geem, Z. W.: Research Commentary: Survival of the Fittest Algorithm or the Novelest Algorithm?. International Journal of Applied Metaheuristic Computing $1(4), 75-79(2010)$

28. Rahman, M. A.: Reliability Analysis of Zigbee based Intra-Vehicle Wireless Sensor Networks. Lecture Notes in Computer Scienc 8435, 103-112 (2014) 